\section{Nexo Técnico Epidemiológico Previdenciário (NTEP): risco das sete atividades econômicas e condições incapacitantes mais frequentes, Brasil, 2000-2016}

\author{
Social Security Epidemiological Technical Nexus \\ (NTEP): risk of seven economic activities and \\ most frequent disabling conditions, Brazil, \\ 2000-2016
}

Paulo Rogério Albuquerque de Oliveira 1,2 Margareth Crisóstomo Portela 3 Heleno Rodrigues Corrêa Filho 4 William Rosa de Souza 5

doi: 10.1590/0102-311X00191119

\section{Resumo}

O Instituto Nacional de Seguro Social do Brasil usa o Nexo Técnico Epidemiológico Previdenciário (NTEP) desde 2007 para associar riscos de ocorrência de incapacidade laboral com classes de atividades econômicas. Este trabalho visou a identificar os principais riscos desse tipo nas empresas brasileiras. Realizamos um estudo de coorte censitária dinâmica, com dados nacionais secundários: o Sistema Único de Benefício (SUB) e o Cadastro Nacional de Informações Sociais (CNIS). O número de identificação do trabalhador (NIT) permitiu vincular a ocorrência de condições incapacitantes (Classificação Internacional de Doenças, 10 a revisão - CID-10) com as atividades econômicas (Classificação Nacional de Atividades Econômicas - CNAE). O NTEP foi estabelecido com os maiores riscos entre as sete classes CNAE mais populosas e sete maiores grupos CID-10 entre os benefícios previdenciários de 2000 a 2016. O Brasil teve no período uma população de empregados segurados de 30.815.310,06 vínculos-médios ao ano com 512.967.233, 15 vínculos-dias. As CNAE mais populosas foram: "comércio varejista - hipermercados", "transporte rodoviário coletivo de passageiros", "bancos múltiplos, com carteira comercial", "abate de suínos e aves", "seleção e agenciamento de mão de obra", "coleta de resíduos não perigosos" e "fabricação de automóveis, camionetas e utilitários". As condições incapacitantes mais prevalentes foram: dorsopatias, traumatismos do punho e da mão, traumatismos do joelho e da perna, transtornos dos tecidos moles, transtornos do humor, artropatias e transtornos neuróticos. Entre as 49 combinações de CID-10 e CNAE estabeleceu-se o NTEP para 27 (55,1\%). O estudo ratifica a acurácia e consistência do NTEP para identificar riscos e frações etiológicas.

Indenização aos Trabalhadores; Licença Médica; Seguro por Invalidez

\author{
Correspondência \\ P. R. A. Oliveira \\ Q 107, Lote 246, Bloco E, apto. 1404, Brasília, DF 71920-540, \\ Brasil. \\ paulorog1966@gmail.com \\ 1 Ministério da Economia, Brasília, Brasil. \\ 2 Universidade Paulista, Brasília, Brasil. \\ ${ }^{3}$ Escola Nacional de Saúde Pública Sergio Arouca, Fundação \\ Oswaldo Cruz, Rio de Janeiro, Brasil. \\ 4 Faculdade de Ciências da Saúde, Universidade de Brasília, \\ Brasília, Brasil. \\ 5 Ministério Público do Trabalho, Goiânia, Brasil.
}




\section{Introdução}

No Brasil, historicamente, por diferentes razões, agravos à saúde do trabalhador ao serem analisados para fins previdenciários frequentemente têm baixa probabilidade de serem vinculados ao trabalho. Há fortes evidências na literatura científica demonstrando extensa subnotificação dos acidentes do trabalho. Em 1990, 12\% dos benefícios eram acidentários e em 2005 caíram para apenas 8\% 1. O sistema de informações acidentário, modelado pelo legislativo brasileiro para a Previdência Social, tem na Comunicação de Acidente do Trabalho (CAT) a sua fonte primária, com fortes indícios de sonegação 2.

A sonegação da CAT está enraizada e demarcada por aspectos econômicos, jurídicos e sociais 3 :

(1) O acidente-doença do trabalho é uma categoria considerada pejorativa e as empresas evitam que dados apareçam nas estatísticas oficiais;

(2) A sua emissão implica o reconhecimento de estabilidade no emprego - um ano de duração a partir do retorno - e isto se contrapõe à liberdade de despedir o trabalhador a qualquer tempo;

(3) Impõe o depósito da contribuição devida de $8 \%$ do salário, em conta do Fundo de Garantia do Tempo de Serviço (FGTS), correspondente ao período de afastamento;

(4) Associa-se ao reconhecimento de fatores de riscos laborais, o que impõe o recolhimento da contribuição específica correspondente ao custeio da aposentadoria especial para os trabalhadores expostos;

(5) Ainda que se constituísse somente em um ato administrativo previdenciário e de sentinela epidemiológica, a CAT emitida pela empresa é tida como palavra final sobre a causalidade acidentária;

(6) A CAT, sob o prisma do empregador, funciona como confissão de culpa com consequências penais, cíveis, previdenciárias e trabalhistas;

(7) O Instituto Nacional de Seguro Social (INSS) tende a condicionar a concessão do benefício acidentário e a prestação de reabilitação profissional à apresentação da CAT, o que imprime a ela valor extraordinário; de um lado isto estimula a subnotificação por parte do empresário e, do outro, ultraja o direito dos empregados;

(8) As doenças do trabalho têm múltiplos fatores etiológicos, que oneram o diagnóstico diferencial, abrindo enorme espaço ao falso negativo (subnotificação);

(9) O afastamento é relacionado ao trabalho ou não? Os serviços médicos contratados pelas empresas tendem a negar a relação ao excluir a contribuinte laboral do espectro dos fatores determinantes, concausais, facilitadores ou desencadeadores do adoecimento, obstaculizando a emissão da CAT, mesmo quando o trabalho é causa suficiente. Isso ocorre ainda mais notadamente quando há motivos econômicos-jurídicos ou a incerteza diagnóstica, ainda que a presunção acidentária esteja prevista na legislação 4;

(10) Na medida em que a CAT é tratada como a fonte primária de informação para a tributação do Seguro Acidente do Trabalho (SAT), sem qualquer flexibilização da captura de casos por outros mecanismos, empresas se veem estimuladas à sua subnotificação, o que termina afetando o seu potencial papel na vigilância epidemiológica da Saúde do Trabalhador.

O Nexo Técnico Epidemiológico Previdenciário (NTEP) foi instituído pela Lei no 11.430, de 26 de dezembro de 2006 , que estabeleceu a possibilidade da perícia médica do INSS considerar caracterizada a natureza acidentária da incapacidade com base na ocorrência de nexo técnico epidemiológico entre o trabalho e o agravo, decorrente da relação entre a atividade da empresa e a condição motivadora da incapacidade elencada na Classificação Internacional de Doenças (CID). Assim, o NTEP foi acrescentado ao rol das espécies da tipologia acidentária de modo a não depender da emissão da CAT 6 .

Ainda em 2007, a Confederação Nacional da Indústria (CNI) ajuizou uma Ação Direta de Inconstitucionalidade ( $A D I$ n n 3.931/2007) contra a instituição do NTEP, que após tramitar por quase 13 anos no Supremo Tribunal Federal (STF) foi julgada, em 17 de abril de 2020, improcedente. O NTEP teve, portanto, a sua constitucionalidade ratificada.

O método tem permitido a captura de relações entre trabalho e saúde antes não contempladas, e estimulado a ampliação do conhecimento acerca destas relações, com o seu mapeamento e quantificação 6,7,8,9,10. Também tem possibilitado a construção de novas hipóteses passíveis de aprofundamento etiológico. O modo como estabelece o nexo epidemiológico previdenciário requer estudos adicionais para a validação e a capacidade de apreensão da realidade dinâmica do trabalho, com vistas à sua aplicação constantemente atualizada. 
Operacionalmente, o NTEP compreende a aferição da morbidade incapacitante produzida pelas empresas empregadoras brasileiras, com base na apuração dos afastamentos relacionados ao trabalho - temporários e permanentes - cobertos pelo INSS, para fins de tributação flexível e presunção acidentária e a identificação de classes de atividades econômicas, segundo a Classificação Nacional de Atividades Econômicas (CNAE) 11, que constituem fatores de risco previdenciário para condições incapacitantes, definidas por grupos diagnósticos da Classificação Internacional de Doenças, 10a revisão (CID-10).

Na perspectiva de apresentação de como o NTEP constrói os estimadores de risco e o fortalecimento de base científica para a sua sustentação, este estudo teve como objetivo identificar as sete classes de atividades do CNAE mais populosas no conjunto das empresas brasileiras, os sete grupos da CID-10 mais frequentes entre os benefícios previdenciários concedidos entre 2000 e 2016, bem como estabelecer entre elas o risco previdenciário e suas frações etiológicas.

\section{Método}

\section{Delineamento}

Temos neste estudo um delineamento do tipo coorte, censitária e dinâmica, iniciada em 1o de janeiro de 2000 e seguida até 31 de dezembro de 2016.

\section{Fontes de dados}

A coorte está baseada em duas fontes de dados institucionais: o Sistema Único de Benefício (SUB) do INSS, e o Cadastro Nacional de Informações Sociais (CNIS) da Secretaria de Previdência Social, ambos administrados pela Dataprev, empresa de processamento de dados da Previdência Social.

O CNIS contém os registros cadastrais das empresas empregadoras, agregando o Cadastro Nacional de Pessoa Jurídica (CNPJ) ao de atividades econômicas (CNAE), bem como aos dados cadastrais de seus empregados, identificados pelo Número de Identificação do Trabalhador (NIT), o que permite marcar a entrada e a saída da coorte.

A variável de exposição é apurada por vínculo-dia a partir do período compreendido entre datas de admissão e dispensa do trabalhador. Cada vínculo empregatício é considerado por NIT se ao menos uma vez ao longo da coorte houve remuneração mensal para este NIT, excluído o período de afastamento por benefício previdenciário. Assim, conta-se um vínculo-dia àquele segurado remunerado que não teve suspensão do contrato de trabalho por motivo de doença/acidente incapacitante.

O SUB é um sistema de informação de grande porte que unifica todos os registros de concessão e manutenção de benefícios operados pelo INSS. O SUB é a origem dos dados da casuística individualizada pelo NIT. Cada benefício é cadastrado no SUB mediante chave-primária, denominada número de benefício (NB), com 10 caracteres numéricos. Entraram na casuística os benefícios cuja Data do Despacho do Benefício (DDB) estava compreendida entre 1o de janeiro de 2000 e 31 de dezembro de 2016. Pelo NB é possível encontrar as espécies de benefícios usadas neste estudo - auxílio-doença, B31; auxílio-doença acidentário, B91; invalidez, B32; e invalidez acidentária, B92, além do diagnóstico (CID-10) incapacitante. O chaveamento entre CNIS e SUB é feito pelo NIT. A Figura 1 exibe o macro fluxo e o processamento das entradas, saídas e a produção de indicadores epidemiológicos para a geração do NTEP. Este trabalho é censitário pelo fato de alcançar toda a população formalmente empregada vinculada à Previdência Social brasileira por intermédio do CNIS.

O adoecimento/incapacitação, variável de desfecho, é expresso pelo diagnóstico, conforme a CID-10, relacionado ao benefício por incapacidade, temporária ou permanente. A CID-10 contempla diferentes níveis de agregação de diagnósticos, que compreendem desde a definição de 12.423 subcategorias identificadas por quatro dígitos à sua organização em termos de 22 grandes capítulos. Dois níveis de agregação intermediários consideram 1.205 categorias diagnósticas truncadas no terceiro dígito e 212 grupos reunindo estas categorias de três dígitos, referidos aqui como CID-10A (CID agrupada) e empregadas no estudo. Por exemplo, o grupo da CID-10A M60-M79 (Tenossinovite e 


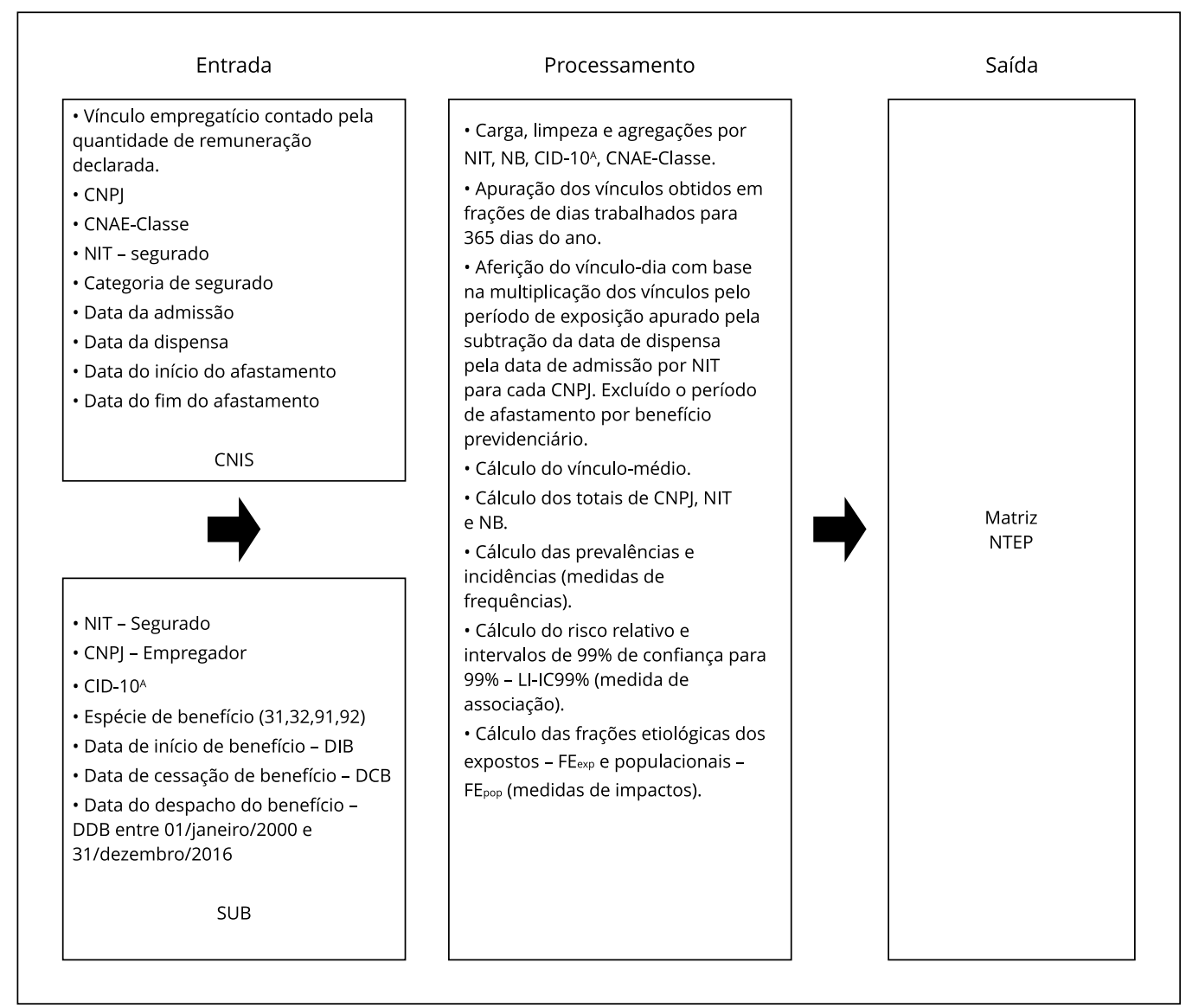

CID: Classificação Internacional de Doenças; CID-10A: CID agrupada; CNAE: Classificação Nacional das Atividades Econômicas; CNIS: Cadastro Nacional da Indústria; CNPJ: Cadastro Nacional de Pessoa Jurídica; NB: número do benefício; NIT: número de identificação do trabalhador; RR: risco relativo; SUB: sistema único de benefício.

Mialgia) inclui 13 categorias diagnósticas designadas por três dígitos e 112 subcategorias diagnósticas designadas por quatro dígitos.

A variável de exposição é definida em termos da atividade econômica desempenhada, classificada com base na CNAE. Esta está estruturada em 21 seções (uma letra), 87 divisões (dois dígitos), 286 grupos (três dígitos), 673 classes (quatro dígitos) e 1.302 subclasses (sete dígitos). Analogamente à abordagem de diagnósticos agrupados, também opta-se aqui pela escolha das atividades econômicas designadas pelas 673 classes do CNAE.

Os microdados utilizados foram oriundos dos computadores de grande porte que hospedam o CNIS e o SUB, sendo extraídos em extensão de arquivo do tipo "txt" como resultado do programa extrator específico preparado e executado pela Dataprev. Esse conjunto de microdados foi repassado formalmente ao primeiro autor deste artigo, via FTP com login/senha deste usuário, com as salvaguardas de confidencialidade. Fez-se uso da plataforma de Business Discovery - QlikView (https:// www.qlik.com/pt-br/products/qlikview), licença livre para a construção da visão geral unificada e 
coerente dos dados baseando-se na indexação e associação de campos, geração das tabelas primárias e tratamento de validação quanto à integridade dos dados.

Conforme já mencionado, a variável NIT foi usada como variável chave para a junção do SUB e CNIS, permitindo a obtenção dos registros de benefícios previdenciários a serem somados segundo a combinação do ano de despacho do benefício, espécie de benefício, classe do CNAE e CID-10A. Na sequência foram feitas as totalizações.

Foram considerados casos novos todas as concessões de benefícios pelo INSS, nas espécies B31 (auxílio-doença previdenciário - doença comum), B32 (aposentadoria de invalidez), B91(auxíliodoença por acidente do trabalho) e B92 (aposentadoria por invalidez por acidente do trabalho) após 15 dias de afastamento, relacionadas a segurados empregados e que cumpriram carência, nos termos da lei previdenciária.

No relacionamento de 212 grupos de CID-10A e 673 classes CNAE, são passíveis de análise 142.676 combinações de condições e atividades econômicas. O recorte neste trabalho contempla os sete grupos da CID-10A mais frequentes entre os benefícios concedidos e as sete classes CNAE mais populosas.

O primeiro autor foi dispensado da obtenção de autorização específica pelo Comitê de Ética por serem dados secundários em uso obrigatório na função que exercia como servidor público, incluindo a obrigação de preservar o segredo para dados identificadores individuais.

\section{Análises}

Parte-se da hipótese nula $\left(\mathrm{H}_{0}\right)$ de que o vínculo empregatício em uma empresa pertencente a uma determinada classe CNAE não constitui fator de risco previdenciário para o trabalhador apresentar desfecho incapacitante por mais de 15 dias em um determinado CID-10A. Ao se rejeitar a hipótese nula $\left(\mathrm{H}_{0}\right)$, assume-se a hipótese alternativa $(\mathrm{Ha})$ de que a classe CNAE constitui fator de risco para a CID-10A.

A prevalência geral (PG) foi obtida pela quantidade-média, por ano, dos CID-10A, dividida pelo vínculo-médio, por ano, de todos os empregados. Da mesma forma, a prevalência por CNAE (PC) foi obtida para o recorte populacional e casuística por classe CNAE. A incidência acumulada entre os expostos (IAEE) foi obtida pelo quociente dos casos novos da classe CNAE pelos seus vínculos-dias, já a incidência acumulada entre os não expostos (IAENE) foi obtida pelo quociente dos casos novos das demais CNAE-Classe pelos seus respectivos vínculos-dias.

Adota-se o risco relativo (RR) como estimador de risco na matriz de contingência $(2 \times 2)$ entre classe CNAE e CID-10A. O grupo exposto (sob teste) é formado por todos os empregados pertencentes à classe CNAE vinculados ao CNIS. O grupo referente é formado por todos os empregados pertencentes às demais classes CNAE. Inserem-se como pertinentes ao CID-10A todos os benefícios associados a categorias CID-10 incapacitantes nele contidos. Não são pertinentes ao CID-10A os benefícios relativos a outras categorias CID-10 ou casos sem benefícios associados.

Por ser um estudo censitário, inferências poderiam ser dispensáveis. Todavia, a ideia de se fazer inferências para a aplicação do método em outros casos fora da coorte é inerente à proposta do NTEP. Assim, são estimados os intervalos de $99 \%$ de confiança (IC99\%) e, de forma conservadora, estabelece-se a existência de NTEP caso o limite inferior do intervalo seja maior do que 1 (LI-IC99\% > 1) 12.

O risco atribuível populacional $\left(\mathrm{RA}_{\mathrm{pop}}\right)$ foi encontrado aplicando-se a fórmula:

$$
R A_{\text {pop }}=(I A E E-I A E N E)
$$

A fração etiológica dos expostos $\left(\mathrm{FE}_{\text {exp }}\right)$ foi calculada como:

$$
F E_{\text {exp }}=\frac{I A E E-I A E N E}{I A E E} \times 100
$$

Estimou-se a fração etiológica populacional $\left(\mathrm{FE}_{\mathrm{pop}}\right)$ com a substituição da IAEE pela incidência na população geral, que é uma média ponderada entre IAEE e IAENE. Tem-se, então, a incidência na população $\left(\mathrm{I}_{\mathrm{pop}}\right)$ assim calculada:

$$
I_{p o p}=[I A E E \times P C]+[\operatorname{IAEE} \times(1-P C)]
$$


$\mathrm{A} \mathrm{FE}_{\mathrm{pop}}$ foi calculada pela seguinte fórmula 13:

$$
F E_{\text {pop }}=\frac{I A E E-I A E N E}{I_{p o p}} \times 100
$$

A Tabela 1 esquematiza em um exemplo como as estatísticas são construídas considerando-se a classe 6422 da CNAE (bancos múltiplos, com carteira comercial) e o grupo F30-F39 da CID-10A (transtornos do humor). Para todas as combinações de classe CNAE e CID-10A consideradas no estudo, as operações foram replicadas, conforme apresentado.

\section{Resultados}

O Brasil apresentou no período entre 2000 e 2016 uma população de segurados empregados de 512.967.233,15 vínculos-dias, com 30.815.310,06 vínculos-médios ao ano. As atividades econômicas mais populosas foram, segundo a CNAE, em ordem decrescente: classe 4711 - Comércio varejista - hipermercados, 861.648,00 vínculos-médios ao ano; classe 4921 - Transporte rodoviário coletivo de passageiros, 442.929,00 vínculos-médios ao ano; classe 6422 - Bancos múltiplos, com carteira comercial, 355.085,00 vínculos-médios ao ano; classe 1012 - Abate de suínos e aves, 198.622,47 vínculos-médios ao ano; classe 7810 - Seleção e agenciamento de mão de obra, 113.745,41 vínculosmédios ao ano; classe 3811 - Coleta de resíduos não perigosos, 86.791,12 vínculos-médios ao ano; e classe 2910 - Fabricação de automóveis, camionetas e utilitários, 80.200,41 vínculos-médios ao ano.

Foram concedidos, nesse período, 37.354 .818 benefícios por incapacidade, dos quais 13.717.463 estão atrelados aos segurados empregados nas espécies B31, B32, B91 e B92. A Figura 2 mostra o universo de benefícios (13.717.463), as perdas (805.197) e a casuística restrita aos segurados empregados para essas espécies que encerram o objeto do estudo (12.912.266).

As entidades mórbidas mais prevalentes foram, em ordem decrescente, por quantidades absolutas de benefícios: M40-M54 - Dorsopatias, 2.200.753; S60-S69 - Traumatismos do punho e da mão, 1.750.813; S80-S89 - Traumatismos do joelho e da perna, 1.515.242; M60-M79 - Transtornos dos tecidos moles, 1.437.903; F30-F39 - Transtornos do humor, 1.106.740; M00-M25 - Artropatias, 1.058.282; e F40-F48 - Transtornos neuróticos, 483.456.

A Tabela 2 apresenta os indicadores epidemiológicos relacionando os sete CID-10A mais prevalentes a cada uma das classes CNAE mais populosas.

Das 49 (100\%) relações entre as sete classes CNAE e os sete CID-10A selecionados, foram observadas 27 associações com NTEP (55,1\%), com base na refutação da hipótese nula com LI-IC99\% > 1. Para $22(44,9 \%)$ combinações de atividade econômica e diagnóstico incapacitante, a hipótese nula não foi rejeitada, assumindo-se a inexistência de NTEP.

O grupo da CID-10A M40-M54 (dorsopatias), apesar de ser o mais prevalente [420,10 (x 100.000)], só apresentou NTEP para as classes 1012 (abate de suínos e aves), 2910 (fabricação de automóveis, camionetas e utilitários), 3811 (coleta de resíduos não perigosos) e 4921 (transporte rodoviário coletivo de passageiros).

O grupo F40-F48 (transtornos neuróticos), por outro lado, sendo o menos prevalente [92,29 (x 100.000)], estabeleceu NTEP com as atividades econômicas designadas pelas classes CNAE 1012 (abate de suínos e aves), 4711 (comércio varejista - hipermercados), 4921 (transporte rodoviário coletivo de passageiros) e 6422 (bancos múltiplos, com carteira comercial). Vale sublinhar que, apesar da estimativa do risco relativo de ocorrência de diagnósticos no grupo F40-F48 (transtornos neuróticos) na classe CNAE 2910 (fabricação de automóveis, camionetas e utilitários) ser 1,04, o LI-IC99\%, de 0,97 , não atendeu o critério para estabelecimento do NTEP.

Observando-se a classe CNAE 4711 (comércio varejista - hipermercados), a mais populosa, notase que apresentou NTEP com os CID-10A F30-F39 (transtornos do humor), F40-F48 (transtornos neuróticos), M60-M79 (transtornos dos tecidos moles) e S60-S69 (traumatismos do punho e da mão). Já a classe 2910 (fabricação de automóveis, camionetas e utilitários), a menos populosa, apresentou NTEP com CID-10A M00-M25 (artropatias), M40-M54 (dorsopatias), M60-M79 (transtornos dos tecidos moles) e S80-S89 (traumatismos do joelho e da perna). 
Tabela 1

Matriz de definição dos grupos (exposto e referente) com indicadores.

\begin{tabular}{lccccc}
\hline & F30-F39 & Demais CID ou sem benefícios & Vínculo-dia & $\begin{array}{c}\text { IAEE } \\
(\mathbf{x ~ 1 0 0 . 0 0 0 )}\end{array}$ & $\begin{array}{c}\text { IAENE } \\
(\mathbf{x} 100.000)\end{array}$ \\
\hline CNAE-classe: 6422 & 26.758 & 328.327 & 5.901 .401 & 453,42 & 2,13 \\
Demais CNAE-classes & 1.078 .982 & 29.380 .243 & 507.065 .833 & & 212,79 \\
Total & 1.105 .740 & 29.708 .570 & 512.967 .234 & & \\
\hline
\end{tabular}

CID: Classificação Internacional de Doenças: CNAE: Classificação Nacional de Atividades Econômicas; IAEE: incidência acumulada entre os expostos; IAENE: incidência acumulada entre os não expostos; IC99\%: intervalo de 99\% de confiança; RR: risco relativo.

\section{Figura 2}

Universo de benefícios com a casuística alvo, as perdas e a casuística objeto do estudo restrita aos segurados empregados para as espécies B31, B32, B91 e B92. Brasil, 2000 a 2016.

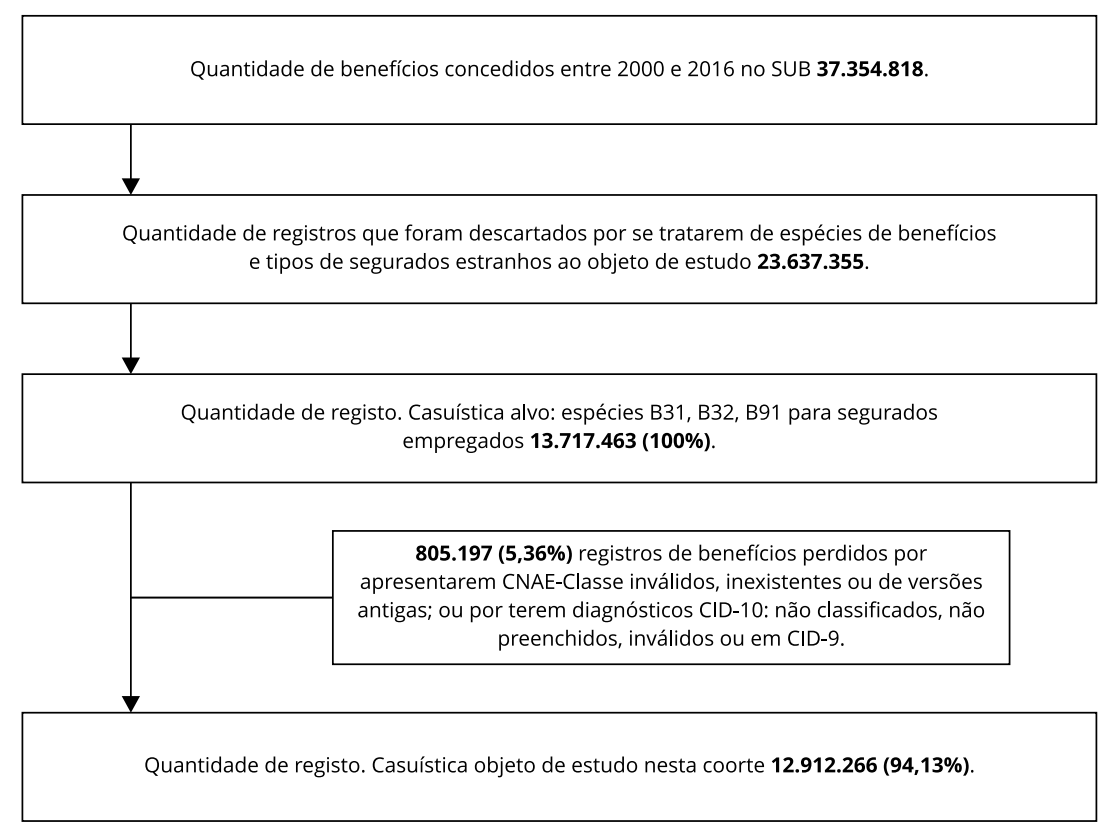

CID: Classificação Internacional de Doenças; CNAE: Classificação Nacional das Atividades Econômicas; SUB: sistema único de benefício.

Há extremos que merecem destaque. A classe CNAE 1012 (abate de suínos e aves) esteve associada com a ocorrência dos sete grupos CID-10A, já a classe 7810 (seleção e agenciamento de mão de obra) não esteve associada a nenhum dos grupos diagnósticos considerados.

Outro destaque relevante é a associação da ocorrência de transtornos neuróticos (grupo CID-10A F40-F48) com a classe CNAE 4921 (transporte rodoviário coletivo de passageiros). Nela observou-se elevada proporção de casos $(6,93 \%)$, maior $\mathrm{RR}(5,30)$, maior $\mathrm{FE}_{\exp }(81,14 \%)$ e maior razão de prevalência $(\mathrm{RP}=4,82)$. 
Tabela 2

Indicadores epidemiológicos relativos à ocorrência de condições incapacitantes (grupo de três dígitos da CID-10) frequentes em atividades econômicas populosas (classe CNAE) entre segurados empregados do Regime Geral da Previdência Social, com base nos vínculos-médios e vínculos-dias. Brasil, 2000 a 2016.

\begin{tabular}{|c|c|c|c|c|c|c|c|c|c|c|}
\hline CID-10A & $\begin{array}{l}\text { Casos } \\
\text { Brasil }\end{array}$ & $\begin{array}{l}\text { Casos } \\
\text { CNAE- } \\
\text { classe }\end{array}$ & $\begin{array}{c}\text { Proporção } \\
\text { de } \\
\text { casos (\%) }\end{array}$ & $\begin{array}{l}\text { Estima- } \\
\text { tiva }\end{array}$ & $\begin{array}{l}\text { RR } \\
\qquad \text { IC99\% }\end{array}$ & $\begin{array}{c}\mathrm{FE}_{\exp } \\
(\%)\end{array}$ & $\begin{array}{c}\mathrm{FE}_{\text {pop }} \\
(\%)\end{array}$ & $\begin{array}{c}\text { Prevalência } \\
\text { CNAE } \\
\text { (x 100.000) }\end{array}$ & $\begin{array}{l}\text { Prevalência } \\
\text { geral } \\
\text { (x 100.000) }\end{array}$ & RP \\
\hline \multicolumn{11}{|l|}{$\begin{array}{l}1012 \text { - Abate de suínos, aves } \\
\text { e outros pequenos animais } \\
\text { [198.622,47 vínculos-médios ao } \\
\text { ano e } 3.203 .768,52 \text { vínculos-dias } \\
\text { (n=0,62\%)] }\end{array}$} \\
\hline $\begin{array}{l}\text { Transtornos do humor } \\
\text { (F30-F39) }\end{array}$ & 1.106 .740 & 19.789 & 1,79 & 2,90 & $2,88-2,92$ & 65,48 & 1,10 & 586,07 & 211,27 & 2,77 \\
\hline $\begin{array}{l}\text { Transtornos neuróticos } \\
\text { (F40-F48) }\end{array}$ & 483.456 & 3.876 & 0,80 & 1,29 & $1,24-1,33$ & 22,24 & 0,03 & 114,79 & 92,29 & 1,24 \\
\hline $\begin{array}{l}\text { Artropatias } \\
\text { (M00-M25) }\end{array}$ & 1.058 .282 & 9.484 & 0,90 & 1,44 & $1,41-1,47$ & 30,50 & 0,12 & 280,88 & 202,02 & 1,39 \\
\hline $\begin{array}{l}\text { Dorsopatias } \\
\text { (M40-M48) }\end{array}$ & 2.200 .753 & 20.606 & 0,94 & 1,50 & $1,48-1,52$ & 33,51 & 0,31 & 610,26 & 420,10 & 1,45 \\
\hline $\begin{array}{l}\text { Transtornos dos tecidos moles } \\
\text { (M60-M79) }\end{array}$ & 1.437 .903 & 32.559 & 2,26 & 3,69 & $3,67-3,70$ & 72,87 & 2,52 & 964,26 & 274,48 & 3,51 \\
\hline $\begin{array}{l}\text { Traumatismos do punho e da } \\
\text { mão (S60-S69) }\end{array}$ & 1.750 .813 & 17.943 & 1,02 & 1,65 & $1,63-1,67$ & 39,30 & 0,34 & 531,40 & 334,21 & 1,59 \\
\hline $\begin{array}{l}2910 \text { - Fabricação de } \\
\text { automóveis, camionetas e } \\
\text { utilitários [80.200,41 vínculos- } \\
\text {-médios ao ano e 1.323.151,34 } \\
\text { vínculos-dias ( } n=0,26 \% \text { )] }\end{array}$ & & & & & & & & & & \\
\hline $\begin{array}{l}\text { Transtornos do humor } \\
\text { (F30-F39) }\end{array}$ & 1.106 .740 & 2.758 & 0,25 & 0,97 & $0,92-1,02$ & $-3,52$ & $-0,01$ & 202,29 & 211,27 & 0,96 \\
\hline $\begin{array}{l}\text { Transtornos neuróticos } \\
\text { (F40-F48) }\end{array}$ & 483.456 & 1.297 & 0,27 & 1,04 & $0,97-1,11$ & 3,86 & 0,00 & 95,13 & 92,29 & 1,03 \\
\hline Artropatias (M00-M25) & 1.058 .282 & 6.686 & 0,63 & 2,46 & $2,43-2,49$ & 59,33 & 0,71 & 490,39 & 202,02 & 2,43 \\
\hline Dorsopatias (M40-M48) & 2.200 .753 & 11.478 & 0,52 & 2,03 & $2,00-1,05$ & 50,67 & 0,86 & 841,86 & 420,10 & 2,00 \\
\hline $\begin{array}{l}\text { Transtornos dos tecidos } \\
\text { moles (M60-M79) }\end{array}$ & 1.437 .903 & 16.936 & 1,18 & 4,61 & $4,59-4,63$ & 78,30 & 4,29 & $1.242,18$ & 274,48 & 4,53 \\
\hline $\begin{array}{l}\text { Traumatismos do punho e da } \\
\text { mão (S60-S69) }\end{array}$ & 1.750 .813 & 4.174 & 0,24 & 0,92 & $0,88-0,97$ & $-8,22$ & $-0,02$ & 306,14 & 334,21 & 0,92 \\
\hline $\begin{array}{l}\text { Traumatismos do joelho e da } \\
\text { perna (S80-S89) }\end{array}$ & 1.515 .242 & 4.901 & 0,32 & 1,25 & $1,22-1,29$ & 20,30 & 0,09 & 359,47 & 289,25 & 1,24 \\
\hline
\end{tabular}

(continua)

O segundo maior RR $(4,61)$ desta coorte ficou para a ocorrência de transtornos de tecidos moles (M60-M79) na classe CNAE 2910 (fabricação de automóveis, camionetas e utilitários), com elevados impactos expressos pela $\mathrm{FE}_{\text {exp }}$ (78,30\%), bem como pela $\mathrm{FE}_{\text {pop }}$ (4,29\%), esta última a maior do estudo.

Em sentido oposto, também é importante notar que a ocorrência de traumatismo de punho e mãos (CID-10A S60-S69) na classe CNAE 6422 (bancos múltiplos, com carteira comercial) foi baixa, apresentando a menor proporção de casos $(0,36 \%)$, menor $\mathrm{RR}(0,31)$ e menor $\mathrm{RP}(0,31)$. 
Tabela 2 (continuação)

\begin{tabular}{|c|c|c|c|c|c|c|c|c|c|c|}
\hline CID-10A & $\begin{array}{l}\text { Casos } \\
\text { Brasil }\end{array}$ & $\begin{array}{l}\text { Casos } \\
\text { CNAE- } \\
\text { classe }\end{array}$ & $\begin{array}{c}\text { Proporção } \\
\text { de } \\
\text { casos (\%) }\end{array}$ & $\begin{array}{c}\text { Estima- } \\
\text { tiva }\end{array}$ & $\begin{array}{l}\text { RR } \\
\qquad \text { IC99\% }\end{array}$ & $\begin{array}{l}\mathrm{FE}_{\exp } \\
(\%)\end{array}$ & $\begin{array}{c}\mathrm{FE}_{\text {pop }} \\
(\%)\end{array}$ & $\begin{array}{c}\text { Prevalência } \\
\text { CNAE } \\
\text { (x 100.000) }\end{array}$ & $\begin{array}{c}\text { Prevalência } \\
\text { geral } \\
\text { (x 100.000) }\end{array}$ & RP \\
\hline \multicolumn{11}{|l|}{$\begin{array}{l}3811 \text { - Coleta de resíduos não } \\
\text { perigosos [86.791,12 vínculos- } \\
\text {-médios ao ano e } 1.410 .419,91 \\
\text { vínculos-dias }(n=0,27 \%)]\end{array}$} \\
\hline $\begin{array}{l}\text { Transtornos do humor } \\
\text { (F30-F39) }\end{array}$ & 1.106 .740 & 2.622 & 0,24 & 0,86 & $0,81-0,91$ & $-16,10$ & $-0,02$ & 177,71 & 211,27 & 0,84 \\
\hline $\begin{array}{l}\text { Transtornos neuróticos } \\
\text { (F40-F48) }\end{array}$ & 483.456 & 1.078 & 0,22 & 0,81 & $0,73-0,89$ & $-23,37$ & $-0,01$ & 73,06 & 92,29 & 0,79 \\
\hline Artropatias (M00-M25) & 1.058 .282 & 5.124 & 0,48 & 1,76 & $1,73-1,80$ & 43,33 & 0,26 & 347,28 & 202,02 & 1,72 \\
\hline Dorsopatias (M40-M48) & 2.200 .753 & 10.433 & 0,47 & 1,73 & $1,70-1,75$ & 42,12 & 0,51 & 707,11 & 420,10 & 1,68 \\
\hline $\begin{array}{l}\text { Transtornos dos tecidos moles } \\
\text { (M60-M79) }\end{array}$ & 1.437 .903 & 5.525 & 0,38 & 1,40 & $1,36-1,43$ & 28,52 & 0,15 & 374,46 & 274,48 & 1,36 \\
\hline $\begin{array}{l}\text { Traumatismos do punho e da } \\
\text { mão (S60-S69) }\end{array}$ & 1.750 .813 & 7.712 & 0,44 & 1,60 & $1,57-1,64$ & 37,68 & 0,32 & 522,69 & 334,21 & 1,56 \\
\hline $\begin{array}{l}\text { Traumatismos do joelho e da } \\
\text { perna ( }(580-589)\end{array}$ & 1.515 .242 & 7.767 & 0,51 & 1,87 & $1,84-1,90$ & 46,49 & 0,46 & 526,42 & 289,25 & 1,82 \\
\hline $\begin{array}{l}4711 \text { - Comércio varejista de } \\
\text { mercadorias em geral, com } \\
\text { predominância de produtos } \\
\text { alimentícios - hipermercados } \\
\text { e supermercados [ } 861.648,00 \\
\text { vínculos-médios ao ano e } \\
13.824 .828,10 \text { vínculos-dias } \\
(n=2,70 \%)]\end{array}$ & & & & & & & & & & \\
\hline $\begin{array}{l}\text { Transtornos do humor } \\
\text { (F30-F39) }\end{array}$ & 1.106 .740 & 39.657 & 3,58 & 1,34 & $1,33-1,36$ & 25,47 & 0,09 & 270,73 & 211,27 & 1,28 \\
\hline $\begin{array}{l}\text { Transtornos neuróticos } \\
\text { (F40-F48) }\end{array}$ & 483.456 & 17.274 & 3,57 & 1,34 & $1,32-1,36$ & 25,25 & 0,04 & 117,93 & 92,29 & 1,28 \\
\hline Artropatias (M00-M25) & 1.058 .282 & 26.843 & 2,54 & 0,94 & $0,92-0,96$ & $-6,43$ & $-0,01$ & 183,25 & 202,02 & 0,91 \\
\hline Dorsopatias (M40-M48) & 2.200 .753 & 52.253 & 2,37 & 0,88 & $0,87-0,89$ & $-13,88$ & $-0,04$ & 356,72 & 420,10 & 0,85 \\
\hline $\begin{array}{l}\text { Transtornos dos tecidos moles } \\
\text { (M60-M79) }\end{array}$ & 1.437 .903 & 58.832 & 4,09 & 1,54 & $1,53-1,55$ & 35,08 & 0,22 & 401,64 & 274,48 & 1,46 \\
\hline $\begin{array}{l}\text { Traumatismos do punho e da } \\
\text { mão (S60-S69) }\end{array}$ & 1.750 .813 & 49.079 & 2,80 & 1,04 & $1,03-1,05$ & 3,96 & 0,01 & 335,06 & 334,21 & 1,00 \\
\hline $\begin{array}{l}\text { Traumatismos do joelho e da } \\
\text { perna (S80-S89) }\end{array}$ & 1.515 .242 & 36.736 & 2,42 & 0,90 & $0,88-0,91$ & $-11,47$ & $-0,03$ & 250,79 & 289,25 & 0,87 \\
\hline
\end{tabular}

(continua) 
Tabela 2 (continuação)

\begin{tabular}{|c|c|c|c|c|c|c|c|c|c|c|}
\hline CID-10A & $\begin{array}{l}\text { Casos } \\
\text { Brasil }\end{array}$ & $\begin{array}{l}\text { Casos } \\
\text { CNAE- } \\
\text { classe }\end{array}$ & $\begin{array}{c}\text { Proporção } \\
\text { de } \\
\text { casos (\%) }\end{array}$ & $\begin{array}{c}\text { Estima- } \\
\text { tiva }\end{array}$ & $\begin{array}{l}\text { RR } \\
\text { IC } 99 \%\end{array}$ & $\begin{array}{l}\mathrm{FE}_{\text {exp }} \\
(\%)\end{array}$ & $\begin{array}{c}\mathrm{FE}_{\text {pop }} \\
(\%)\end{array}$ & $\begin{array}{c}\text { Prevalência } \\
\text { CNAE } \\
\text { (x 100.000) }\end{array}$ & $\begin{array}{c}\text { Prevalência } \\
\text { geral } \\
\text { (x 100.000) }\end{array}$ & $\mathbf{R P}$ \\
\hline $\begin{array}{l}4921 \text { - Transporte rodoviário } \\
\text { coletivo de passageiros, com } \\
\text { itinerário fixo, municipal e } \\
\text { em região metropolitana } \\
\text { [442.929,00 vínculos-médios ao } \\
\text { ano e } 7.100 .234,00 \text { vínculos-dias } \\
\text { ( } n=1,38 \%)]\end{array}$ & & & & & & & & & & \\
\hline $\begin{array}{l}\text { Transtornos do humor } \\
\text { (F30-F39) }\end{array}$ & 1.106 .740 & 35.386 & 3,20 & 2,35 & $2,34-2,37$ & 57,50 & 0,63 & 469,95 & 211,27 & 2,22 \\
\hline $\begin{array}{l}\text { Transtornos neuróticos } \\
\text { (F40-F48) }\end{array}$ & 483.456 & 33.481 & 6,93 & 5,30 & $5,29-5,32$ & 81,14 & 1,88 & 444,65 & 92,29 & 4,82 \\
\hline Artropatias (M00-M25) & 1.058 .282 & 19.155 & 1,81 & 1,31 & $1,29-1,33$ & 23,86 & 0,08 & 254,39 & 202,02 & 1,26 \\
\hline Dorsopatias (M40-M48) & 2.200 .753 & 63.621 & 2,89 & 2,12 & $2,11-2,13$ & 52,85 & 0,94 & 844,92 & 420,10 & 2,01 \\
\hline $\begin{array}{l}\text { Transtornos dos tecidos } \\
\text { moles (M60-M79) }\end{array}$ & 1.437 .903 & 17.307 & 1,20 & 0,87 & $0,85-0,89$ & $-15,21$ & $-0,03$ & 229,85 & 274,48 & 0,84 \\
\hline $\begin{array}{l}\text { Traumatismos do punho e da } \\
\text { mão (S60-S69) }\end{array}$ & 1.750 .813 & 22.331 & 1,28 & 0,92 & $0,90-0,94$ & $-8,64$ & $-0,02$ & 296,57 & 334,21 & 0,89 \\
\hline $\begin{array}{l}\text { Traumatismos do joelho e da } \\
\text { perna ( } \mathrm{S} 80-\mathrm{S} 89 \text { ) }\end{array}$ & 1.515 .242 & 20.807 & 1,37 & 0,99 & $0,97-1,01$ & $-0,81$ & $-0,00$ & 276,33 & 289,25 & 0,96 \\
\hline $\begin{array}{l}6422 \text { - Bancos múltiplos, com } \\
\text { carteira comercial [355.085,00 } \\
\text { vínculos-médios ao ano e } \\
5.901 .400,55 \text { vínculos-dias } \\
(n=1,15 \%)]\end{array}$ & & & & & & & & & & \\
\hline $\begin{array}{l}\text { Transtornos do humor } \\
\text { (F30-F39) }\end{array}$ & 1.106 .740 & 26.758 & 2,42 & 2,13 & $2,11-2,15$ & 53,03 & 0,50 & 443,27 & 211,27 & 2,10 \\
\hline $\begin{array}{l}\text { Transtornos neuróticos } \\
\text { (F40-F48) }\end{array}$ & 483.456 & 19.829 & 4,10 & 3,67 & $3,66-3,69$ & 72,79 & 0,87 & 328,49 & 92,29 & 3,56 \\
\hline Artropatias (M00-M25) & 1.058 .282 & 7.607 & 0,72 & 0,62 & $0,59-0,65$ & $-60,75$ & $-0,05$ & 126,02 & 202,02 & 0,62 \\
\hline Dorsopatias (M40-M48) & 2.200 .753 & 14.816 & 0,67 & 0,58 & $0,56-0,60$ & $-71,71$ & $-0,10$ & 245,44 & 420,10 & 0,58 \\
\hline $\begin{array}{l}\text { Transtornos dos tecidos moles } \\
\text { (M60-M79) }\end{array}$ & 1.437 .903 & 42.517 & 2,96 & 2,62 & $2,60-2,63$ & 61,80 & 1,13 & 704,34 & 274,48 & 2,57 \\
\hline $\begin{array}{l}\text { Traumatismos do punho e da } \\
\text { mão (S60-S69) }\end{array}$ & 1.750 .813 & 6.237 & 0,36 & 0,31 & $0,27-0,34$ & $-225,54$ & $-0,07$ & 103,32 & 334,21 & 0,31 \\
\hline $\begin{array}{l}\text { Traumatismos do joelho e da } \\
\text { perna ( }(580-589)\end{array}$ & 1.515 .242 & 8.575 & 0,57 & 0,49 & $0,46-0,52$ & $-104,49$ & $-0,07$ & 142,05 & 289,25 & 0,49 \\
\hline
\end{tabular}

(continua) 
Tabela 2 (continuação)

\begin{tabular}{|c|c|c|c|c|c|c|c|c|c|c|}
\hline CID-10A & $\begin{array}{l}\text { Casos } \\
\text { Brasil }\end{array}$ & $\begin{array}{l}\text { Casos } \\
\text { CNAE- } \\
\text { classe }\end{array}$ & $\begin{array}{c}\text { Proporção } \\
\text { de } \\
\text { casos (\%) }\end{array}$ & $\begin{array}{c}\text { Estima- } \\
\text { tiva }\end{array}$ & $\begin{array}{l}\text { RR } \\
\qquad \text { IC99\% }\end{array}$ & $\begin{array}{l}\mathrm{FE}_{\exp } \\
(\%)\end{array}$ & $\begin{array}{l}\mathrm{FE}_{\text {pop }} \\
(\%)\end{array}$ & $\begin{array}{c}\text { Prevalência } \\
\text { CNAE } \\
\text { (x 100.000) }\end{array}$ & $\begin{array}{c}\text { Prevalência } \\
\text { geral } \\
\text { (x 100.000) }\end{array}$ & $\mathbf{R P}$ \\
\hline \multicolumn{11}{|l|}{$\begin{array}{l}7810 \text { - Seleção e agenciamento } \\
\text { de mão de obra [113.745,41 } \\
\text { vínculos-médios ao ano e } \\
1.896 .327,65 \text { vínculos-dias } \\
(n=0,37 \%)]\end{array}$} \\
\hline $\begin{array}{l}\text { Transtornos do humor } \\
\text { (F30-F39) }\end{array}$ & 1.106 .740 & 2.990 & 0,27 & 0,73 & $0,68-0,78$ & $-36,97$ & $-0,04$ & 154,63 & 211,27 & 0,73 \\
\hline $\begin{array}{l}\text { Transtornos neuróticos } \\
\text { (F40-F48) }\end{array}$ & 483.456 & 1.143 & 0,24 & 0,64 & $0,56-0,72$ & $-56,57$ & $-0,02$ & 59,11 & 92,29 & 0,64 \\
\hline Artropatias (M00-M25) & 1.058 .282 & 3.110 & 0,29 & 0,79 & $0,75-0,84$ & $-25,89$ & $-0,03$ & 160,83 & 202,02 & 0,80 \\
\hline Dorsopatias (M40-M48) & 2.200 .753 & 6.164 & 0,28 & 0,76 & $0,72-0,79$ & $-32,11$ & $-0,08$ & 318,77 & 420,10 & 0,76 \\
\hline $\begin{array}{l}\text { Transtornos dos tecidos moles } \\
\text { (M60-M79) }\end{array}$ & 1.437 .903 & 4.017 & 0,28 & 0,76 & $0,71-0,80$ & $-32,45$ & $-0,05$ & 207,74 & 274,48 & 0,76 \\
\hline $\begin{array}{l}\text { Traumatismos do punho e da } \\
\text { mão (S60-S69) }\end{array}$ & 1.750 .813 & 5.721 & 0,33 & 0,88 & $0,85-0,92$ & $-13,18$ & $-0,03$ & 295,86 & 334,21 & 0,89 \\
\hline $\begin{array}{l}\text { Traumatismos do joelho e da } \\
\text { perna (S80-S89) }\end{array}$ & 1.515 .242 & 4.742 & 0,31 & 0,85 & $0,81-0,88$ & $-18,19$ & $-0,04$ & 245,23 & 289,25 & 0,85 \\
\hline
\end{tabular}

CID-10A: Classificação Internacional de Doenças agrupada, 10a revisão; CNAE: Classificação Nacional das Atividades Econômicas; $\mathrm{FE}_{\text {exp }}$ : fração etiológica dos expostos; $\mathrm{FE}_{\mathrm{pop}}$ : fração etiológica populacional; RR: risco relativo.

\section{Discussão}

Neste estudo, faz-se um recorte nas 142.676 combinações de condições incapacitantes e atividades econômicas, definidas, respectivamente, pela CID-10A e CNAE, para focar nas combinações envolvendo as sete classes CNAE mais populosas e os sete grupos CID-10A mais frequentes na casuística. A seleção, em termos gerais, é bastante expressiva e o conjunto de atividades econômicas contemplado parece abrangente do ponto de vista ambiental.

Os resultados apresentados reforçam a acurácia da metodologia do NTEP, pois confirmam em ambos os sentidos, afirmando ou negando NTEP, relações etiológicas reportadas na literatura científica $14,15,16,17,18,19,20$.

O achado, por exemplo, de associação da atividade econômica "abate de suínos e aves" com os sete grupos CID-10A considerados é compatível com a concentração de fatores de riscos químicos, físicos, biológicos, mecânicos, psíquicos e ergonômicos nesta indústria 19. Por outro lado, a não identificação de associação da atividade econômica "seleção e agenciamento de mão de obra" parece refletir uma dispersão de fatores de riscos 20.

Conquanto a "prevalência" não tenha o condão de estimar risco, a RP mostrou-se neste estudo como preditor de risco, convergindo com o NTEP, evidenciando paralelismo consistente entre ambos.

As tabelas de contingência estudadas neste relato demonstram relações apropriadas para a análise segundo o modelo caso-coorte. Os casos atendem as definições dos grupos CID-10. A coorte censitária contém todos os outros elementos definidores de exposição às atividades econômicas, que também incluem pessoas incapacitadas nos grupos CID-10 embora não expostas. Portanto, este desenho analítico permite avaliar tanto razões de prevalência quanto risco relativo, fração etiológica de exposição e fração etiológica populacional. Estão presentes na coorte todas as pessoas do Brasil que receberam benefícios de afastamento por incapacidade no mercado formal brasileiro no período. Os intervalos de confiança são adequados para projetar os riscos sobre populações futuras submetidas às mesmas exposições no trabalho típico das classes de atividades econômicas analisadas. 
A proposição do NTEP preencheu uma lacuna relevante na possibilidade de atribuição de condições incapacitantes às atividades laborais, em cenários de muitos conflitos de interesses e problemas no preenchimento adequado da CAT. Perante o INSS, para fins de requerimento e habilitação de benefício por incapacidade, deve-se registrar o diagnóstico incapacitante de acordo com a CID-10. Esse registro é de responsabilidade profissional do médico que presta o atendimento, sendo exigido para a concessão de benefício, seja laboral ou não. Esse processo não padece dos vícios de subjetividade administrativa da CAT, uma vez que independe da comunicação da empresa. Se o segurado for acometido de uma doença-lesão e esta implicar a incapacidade para o exercício de sua atividade, o benefício será concedido pela Previdência Social, independentemente de qualquer comunicação da empresa.

A utilização da CID-10A do benefício incapacitante, em vez da CAT, como variável de desfecho clínico, articula elemento primário idôneo o suficiente para estabelecer o perfil de morbidade previdenciário dos empregados brasileiros, mantém a abordagem clínica individual, agregando um instrumental de saúde coletiva dado pela epidemiologia que alcança os ambientes de trabalho insalubres e possibilita descrever perfis coletivamente 21 . Esse dado primário (CID) tem por características: imunidade à sonegação da empresa pois sempre existirá se houver incapacidade; quem prescreve é o profissional médico e independe de declaração da empresa; independe do desejo/poder do empregador sobre a informação; está intrinsecamente relacionado à incapacidade laboral e à entidade mórbida; ativa a responsabilidade dos médicos avaliadores; é de notificação compulsória para casos previstos em lei; é compartilhado por outros bancos de dados de saúde pública e coexiste nas seguradoras privadas e no SUS; permite estabelecer e tem, em alguns casos, associação etiológica com ambientes do trabalho; e ativa as normas de ética dos Conselhos dos Profissionais da área de saúde.

Neste estudo, a variável desfecho "condição incapacitante", designada pela CID-10A, foi considerada no sentido de possibilitar um balanço entre especificidade e sensibilidade. Trabalhar com 1.205 categorias diagnósticas seria muito específico e pouco sensível, por outro lado, haveria o oposto se fossem considerados os 22 capítulos. A CID-10A acomoda esses extremos de sensibilidade e especificidade em um ponto intermediário.

No que concerne à variável de exposição, a CNAE, além de uma classificação, configura a figura ontológica isomórfica de característica sistêmica, integrada e multidisciplinar, e possibilita a montagem de um painel epidemiológico de morbidade capaz de nortear a formulação de políticas públicas em saúde, com foco no meio ambiente do trabalho, ao definir amplo conjunto de representações, em que se destacam as sociais, epidemiológicas, econômicas e jurídicas.

A afirmação sobre isomorfia ontológica se depreende da dimensão social que a classe CNAE assume ante os indivíduos em empresas (CNPJ) que comungam, convivem, concorrem, compram, vendem, apreçam, contratam, escrituram, licitam, alienam, participam do mercado bursátil, terceirizam, empregam, protegem e adoecem de maneira verossimilhante. Reproduzem práticas multidimensionais de representações plurais. Segundo a teoria burocrática organizacional de Weber 22, essa homogeneidade das empresas, aqui representada pela classe CNAE, decorre da reprodutibilidade de formas e práticas verossímeis comuns que, nos dizeres de Dimaggio \& Powell 23, estão submetidas à Gaiola de Ferro do Isomorfismo Institucional, segundo a racionalidade organizacional weberiana. Práticas verossímeis comuns e reprodutibilidade fazem com que as organizações dentro da mesma classe CNAE estejam mais orientadas à homogeneidade que à competição ou à diversidade, ainda que haja o diferencial competitivo. $\mathrm{O}$ isomorfismo é entendido como o processo de restrição que força uma unidade organizacional em uma população a se assemelhar às outras unidades que enfrentam o mesmo conjunto de condições ambientais, sendo de natureza tripla: coercitivo, mimético e normativo 24 .

$\mathrm{O}$ isomorfismo coercitivo deriva das influências políticas e do problema da legitimidade, decorrentes de pressões formais e informais que sofrem de outras organizações da mesma classe CNAE e das quais dependem, bem como das expectativas culturais da sociedade em que as organizações atuam; o mimético aparece como respostas padronizadas às incertezas. Nem todo isomorfismo deriva da autoridade coercitiva, uma vez que a incerteza constitui poderosa força que encoraja a imitação a outras organizações ditas modelos (benchmarking), notadamente em cenários nos quais as tecnologias de gestão são pouco compreendidas, de soluções pouco nítidas, ou quando se veem frente a problemas de causas ambíguas. O normativo é associado à profissionalização, assim entendida a luta coletiva de membros de uma profissão para definir as condições e os métodos de seu trabalho, para controlar a produção e os produtores, bem como para estabelecer uma base cognitiva e legitimação para a auto- 
nomia da profissão. Segundo essa cognição, um grupo de organizações (classe CNAE) emerge como um campo e suscita um paradoxo: atores racionais tornam suas organizações cada vez mais similares na medida em que tentam transformá-las.

Depreende-se o poder que a classe CNAE tem como construto teórico para assumir o caráter de variável sintética de múltiplas representações e dimensões, com potencial gnosiológico e heurístico. Neste estudo, confere-se à classe CNAE atributos de representação social, epidemiológica e jurídica que a sustentam. Combinando o aspecto ontológico à isomorfia de tripla dimensão, considera-se a classe CNAE como variável sintética, potente e conglobante, de exposição para a estimativa de risco previdenciário.

Registre-se que o NTEP não é um estimador de risco sindrômico 25 ou etiológico, mas apenas e tão somente um estimador de risco previdenciário, dada a natureza estritamente previdenciária da população e da casuística. Por conseguinte, constitui uma impropriedade técnica suscitar objeção contra essa metodologia argumentando que muitas relações não têm explicação etiológica, notadamente aquelas do tipo crônicas, como transtornos mentais, distúrbios osteomusculares e que, portanto, tais relações são espúrias, ou ainda, que são apenas associações estatísticas. É igualmente impróprio arrazoar que só seria possível estabelecer o nexo de causalidade para determinados desfechos clínicos mediante o conhecimento prévio dos mecanismos fisiopatológicos e anátomo-clínicos das relações, assim como a história natural da doença que referenciasse os postulados de causalidade de Hill 26; precipuamente, a força associativa e a plausibilidade. Não se cogita, e não constitui objeto do método desenvolvido, usar técnicas de raciocínio diagnóstico hipotético-dedutivo ou reconhecimento de padrão (as duas mais utilizadas) para perquirir diagnósticos sindrômicos combinados com os diagnósticos etiológicos. O NTEP é um estimador de risco previdenciário e um importante ensejador de suspeitas etiológicas.

Decompondo a sigla NTEP, temos a estrita natureza deste constructo. Nexo sugere uma associação causal (tendente a) 27 . Técnico porque decorre do conhecimento humano acumulado nas disciplinas que fazem interseção com o objeto sob observação. Epidemiológico porque o estudo ocorre sobre populações, cujas análises são feitas por diferenças estatisticamente significativas para determinados desfechos, seguindo padrões metodológicos cientificamente aceitáveis. Previdenciário porque a população e a casuística alcançam os trabalhadores vinculados ao Regime Geral de Previdência Social do Brasil (RGPS), na qualidade de segurado-empregado, inscritos no universo das empresas empregadoras, no âmbito de cada classe CNAE.

Existem limitações para as relações epidemiológicas encontradas neste estudo de coorte censitária e dinâmica nacional entre a exposição a classes de atividade econômica e a possibilidade de existirem riscos de ocorrência elevada em determinados grupos de agravos da CID-10. Um limite deste estudo é que o prazo efetivo entre a exposição presumida e o evento incapacitante é variável entre frações de um ano e décadas de início do trabalho em cada atividade segundo cada trabalhador registrado. Outro limite é que todos os diagnósticos dependem estritamente da avaliação médico-pericial que os reconhece, documenta e insere no sistema de benefícios por incapacidade laboral. A validação dos diagnósticos emitidos por médicos peritos extrapola a pretensão deste estudo. Outra limitação é que as relações de ocorrências epidemiológicas não significam afirmar causalidade. A limitação da leitura dos riscos é que eles antecedem e não sucedem os estudos de causalidade. O NTEP é utilizado como estimador de risco previdenciário e jamais pretendeu operar como estimador de risco sindrômico, clínico ou etiológico. Identificar riscos implica a necessidade de estudar e validar modelos causais pertinentes em outros contextos científicos. É na magnitude dos riscos previdenciários detectados que reside a força do estudo da coorte do NTEP. 


\section{Colaboradores}

P. R. A. Oliveira contribuiu na concepção e projeto, produção, análise e interpretação dos dados, redação do artigo, considerando todos os aspectos de exatidão e integridade da obra, bem como procedimento de revisão final. M. C. Portela colaborou na análise e interpretação dos dados, revisão crítica do conteúdo intelectual e aprovação final da versão a ser publicada. H. R. Corrêa Filho contribuiu na interpretação dos dados, revisão crítica do conteúdo intelectual e aprovação final da versão a ser publicada. W. R. Souza contribuiu na produção, tratamento e análise dos dados, revisão crítica do conteúdo intelectual e aprovação final da versão a ser publicada.

\section{Informações adicionais}

ORCID: Paulo Rogério Albuquerque de Oliveira (0000-0002-4569-6684); Margareth Crisóstomo Portela (0000-0002-9858-9276); Heleno Rodrigues Corrêa Filho (0000-0001-8056-8824); William Rosa de Souza (0000-0002-8915-4895).

\section{Agradecimentos}

Agradecemos à Escola Nacional de Saúde Pública Sergio Arouca, Fundação Oswaldo Cruz, pela oportunidade de disseminação científica.

\section{Referências}

1. Subsecretaria de Regime Geral de Previdência Social, Secretaria de Previdência, Ministério da Fazenda. Anuário estatístico da previdência social - 2016. http://sa.previdencia.gov.br/ site/2018/02/Apresenta\%C3\%A7\%C3\%A3oAlexandre-Ziole-AEPS-2016-003.pdf (acessado em 19/Ago/2020).

2. Santana V, Nobre L, Waldvogel BC. Acidentes de trabalho no Brasil entre 1994 e 2004: uma revisão. Ciênc Saúde Colet 2005; 10:841-55.

3. Lucca SR, Fávero M. Os acidentes do trabalho no Brasil - algumas implicações de ordem econômica, social e legal. Rev Bras Saúde Ocup 1994; 22:7-14.

4. Brasil. Lei no 8.213, de 24 de julho de 1991. Dispõe sobre os Planos de Benefícios da Previdência Social e dá outras providências. Diário Oficial da União 1991; 25 jul.

5. Brasil. Lei no 11.430 , de 26 de dezembro de 2006. Altera as Leis nos 8.213, de 24 de julho de 1991, e 9.796, de 5 de maio de 1999, aumenta o valor dos benefícios da previdência social; e revoga a Medida Provisória no 316, de 11 de agosto de 2006; dispositivos das Leis nos 8.213, de 24 de julho de 1991, 8.444, de 20 de julho de 1992, e da Medida Provisória no 2.187-13, de 24 de agosto de 2001; e a Lei no 10.699, de 9 de julho de 2003. Diário Oficial da União 2006; 27 dez.

6. Almeida PCA, Barbosa-Branco A. Acidentes de trabalho no Brasil: prevalência, duração e despesa previdenciária dos auxílios-doença. Rev Bras Saúde Ocup 2011; 36:195-207.

7. Todeschini R, Codo W. Uma revisão crítica da metodologia do Nexo Técnico Epidemiológico Previdenciário (NTEP). Rev Baiana Saúde Pública 2013 ; 37:486-500.

8. Vieira ER, Albuquerque-Oliveira PR, BarbosaBranco A. Work disability benefits due to musculoskeletal disorders among Brazilian private sector workers. BMJ Open 2011; 1:e000003.

9. Ildefonso SAG, Barbosa-Branco A, Albuquerque-Oliveira PR. Prevalência de benefícios de seguridade social temporária devido a doença respiratória no Brasil. J Bras Pneumol 2009; 35:44-53.

10. Santana VS, Villaveces A, Bangdiwala SI, Runyan CW, Albuquerque-Oliveira PR. Workdays lost due to occupational injuries among young workers in Brazil. Am J Ind Med 2012; 55:91725.

11. Instituto Brasileiro de Geografia e Estatística. Comissão Nacional de Classificação de Atividades Econômicas - Concla. https://concla. ibge.gov.br/busca-online-cnae.html?view =es trutura (acessado em 22/Ago/2020).

12. Morris JA, Gardner MJ. Calculating confidence intervals for relative risks (odds ratios) and standardized ratios and rates. Br Med J (Clin Res Ed) 1988; 296:1313-6.

13. Rezende LFM, Eluf-Neto J. Fração atribuível populacional: planejamento de ações de prevenção de doenças no Brasil. Rev Saúde Pública $2016 ; 50: 30$. 
14. Leigh JP, Miller TR. Occupational illnesses within two national data sets. Int J Occup Environ Health 1998; 4:99-113.

15. Leigh JP, Miller TR. Job-related diseases and occupations within a large workers' compensation data set. Am J Ind Med 1998; 33:197211.

16. Anderson VP, Schulte PA, Sestito J, Linn H, Nguyen LS. Occupational fatalities, injuries, illnesses, and related economic loss in the wholesale and retail trade sector. Am J Ind Med 2010; 53:673-85.

17. Centers for Disease Control and Prevention. Workers' compensation claims for musculoskeletal disorders among wholesale and retail trade industry workers - Ohio, 20052009. MMWR Morb Mortal Wkly Rep 2013; 62:437-42.

18. Anderson VP, Schulte PA, Novakovich J, Pfirman D, Bhattacharya A. Wholesale and retail trade sector occupational fatal and nonfatal injuries and illnesses from 2006 to 2016: implications for intervention. Am J Ind Med 2020; 63:121-34.

19. Dal Magro MLP, Coutinho MC, Moré CLOO. Relações de poder na atenção à saúde do trabalhador formal: o caso da indústria de abate e processamento de carnes. Rev Bras Saúde Ocup 2016; 41:e4.

20. Lourenço EAS. Terceirização: a derruição de direitos e a destruição da saúde dos trabalhadores. Serv Soc Soc 2015; 123:447-75.
21. Facchini LA. Uma contribuição da epidemiologia: o modelo de determinação social aplicado à saúde do trabalhador. In: Buschinelli JTP, Rocha LE, Rigotto RM, organizadores. Isto é trabalho de gente? Vida, doença e trabalho no Brasil. Petrópolis: Editora Vozes; 1994. p. 17886.

22. Weber M. História geral da economia. São Paulo: Mestre Jou; 1968

23. Dimaggio PJ, Powell WW. The iron cage revisited: institutional isomorphism and collective rationality in organizational fields. Am Sociol Rev 1983; 48:147-60.

24. Rosseto CR, Rosseto AM. Teoria institucional e dependência de recursos na adaptação organizacional: uma visão complementar. RAE Eletrônica 2005; 4(1). http://www. scielo.br/scielo.php?script $=$ sci_arttext $\&$ pi $\mathrm{d}=\mathrm{S} 1676-56482005000100010 \& \operatorname{lng}=$ en\&nrm $=$ iso\&tlng $=$ pt.

25. Biselli PJ, Atta JA. Diagnóstico sindrômico. Rev Med (São Paulo) 2005; 84:95-101.

26. Hill AB. The environment and disease: association or causation. Proc R Soc Med 1965; 58:295-300.

27. Barata RB. Causalidade e epidemiologia. Hist Ciênc Saúde-Manguinhos 1997; 4:31-49. 


\section{Abstract}

The Brazilian National Social Security Institute has used the Social Security Epidemiological Technical Nexus (NTEP) since 2007 to associate risks of the occurrence of work-related disability to classes of economic activities. This study aimed to identify the main risks of this type in Brazilian companies. We conducted a dynamic census cohort study with secondary national data from the Single Benefit System (SUB) and the National Registry of Social Information (CNIS). The Worker's Identification Number (NIT) allowed linking the occurrence of disabling conditions (International Classification of Diseases, $10^{\text {th }}$ revision - ICD-10) to economic activities (National Classification of Economic Activities, CNAE). The NTEP was established with the highest risks between the seven most populous CNAE classes and seven largest ICD-10 among social security benefits from 2000 to 2016. During the period, Brazil had entitled employees with 30,815,310.06 employment contracts per year with 512,967,233.15 contract days. The most populous CNAE were: "retail commerce - hypermarkets", "roadway collective passenger transportation", "multipurpose banks with commercial portfolios", "pork and poultry slaughtering", "job selection and hiring", "nonhazardous waste collection", and "automobile, pickup truck, and utility vehicle manufacturing". The most prevalent disabling conditions were: back disorders, wrist and hand injuries, knee and leg injuries, soft tissue disorders, mood disorders, joint disorders, and neurotic disorders. Among the $49 \mathrm{com}$ binations of ICD-10 and CNAE, the NTEP was established for $27(55.1 \%)$. The study corroborates the accuracy and consistency of NTEP for identifying risks and etiological fractions.

Workers' Compensation; Sick Leave; Disability Insurance

\section{Resumen}

El Instituto Nacional de Seguro Social de Brasil usa el Nexo Técnico Epidemiológico de la Seguridad Social (NTEP) desde 2007, para asociar riesgos de ocurrencia de incapacidad laboral con clases de actividades económicas. Este estudio tuvo como meta identificar los principales riesgos de este tipo en empresas brasileñas. Realizamos un estudio de cohorte censal dinámica, con datos nacionales secundarios del Sistema Único de Beneficio (SUB), así como del Registro Nacional de Información Social (CNIS). El número de identificación del trabajador (NIT) permitió vincular la ocurrencia de condiciones incapacitantes (Clasificación Internacional de Enfermedades, 10a edición - CIE-10) con las actividades económicas (Clasificación Nacional de Actividades Económicas CNAE). El NTEP fue establecido con los mayores riesgos entre las siete clases CNAE más pobladas y siete grupos mayores CIE-10 entre los beneficios de seguridad social de 2000 a 2016. Brasil tuvo durante este período una población de empleados asegurados de 30.815.310,06 vínculos-medios al año con 512.967.233, 15 vínculos-dias. Las CNAE más pobladas fueron: "comercio minorista - hipermercados", "transporte por carretera colectivo de pasajeros", "bancos múltiples, con cartera comercial", "matadero de porcino y aves", "selección $y$ contratación de mano de obra", "recogida de residuos no-peligrosos" y "fabricación de automóviles, camionetas y utilitarios". Las condiciones incapacitantes más prevalentes fueron: dorsopatías, traumatismos del puño y de la mano, traumatismos de la rodilla y de la pierna, trastornos de los tejidos blandos, trastornos del humor, artropatías y trastornos neuróticos. Entre las 49 combinaciones de CIE-10 y CNAE se estableció el NTEP para 27 $(55,1 \%)$. El estudio ratifica la precisión y consistencia del NTEP para identificar riesgos y fracciones etiológicas.

Indemnización para Trabajadores; Ausencia por Enfermedad; Seguro por Discapacidad
Recebido em 30/Set/2019

Versão final reapresentada em 01/Set/2020

Aprovado em 28/Set/2020 